\title{
¿Interés público o interés publicado? La regulación de medios argentinos en la prensa económica
}

\author{
Public interest or published interest? \\ Argentine media regulation in economic press
}

\author{
Nadia Koziner \\ Consejo Nacional de Investigaciones Científicas y Técnicas (CONICET) \\ Universidad Nacional de Quilmes (UNQ), Argentina \\ nadiakoziner@gmail.com \\ https://orcid.org/0000-0003-2834-4799
}

\begin{abstract}
Resumen
El Poder Legislativo argentino aprobó la Ley Nº 26522 de Servicios de Comunicación Audiovisual (LSCA) el 10 de octubre de 2009. Esta puso en cuestión la histórica relación entre los objetivos de orden político, sociocultural y económico que habían orientado la regulación y propuso una nueva noción de 'interés público'. El derrotero que siguió el debate y la sanción de la Ley ocupó un lugar significativo en la agenda mediática, aunque fue definido desde marcos particulares que formularon diagnósticos, evaluaciones y prescripciones. Analizar el tratamiento periodístico de este asunto cobra importancia por cuanto implicó los intereses del ámbito del cual los propios forman parte. Además, se los considera coconstructores públicamente destacados de realidad social, por lo cual colaboraron con la producción de los relatos del debate acerca de la Ley. Este trabajo analiza los frames noticiosos de la LSCA en los periódicos económicos argentinos, observa la evolución de dichos encuadres a lo largo del debate y sanción de la norma y compara las maneras en que los matutinos definieron los aspectos puestos en discusión. A partir del análisis de contenido cuantitativo, se identifican tres frames en tensión: 'disputa política e institucional, 'interés púbico sociocultural' e 'interés público empresario', cuyo comportamiento varió según el matutino y registró fluctuaciones a lo largo del período estudiado.
\end{abstract}

\section{Palabras clave}

Prensa, Argentina, regulación, encuadres, interés público, información.

Forma sugerida de citar: Koziner, N. (2021). ¿Interés público o interés publicado? La regulación de medios argentinos en la prensa económica. Univeristas, 34, pp. 155-176. 


\begin{abstract}
The Argentine Legislative Power passed Law No. 26522 on Audiovisual Communication Services (LACS) on October 10, 2009. It called into question the historical relationship among the political, socio-cultural and economic objectives that guided the regulation and represented a new notion of 'public interest'. The process of debate and approval of the Law occupied a significant space on the media agenda, although it was defined from particular frames that formulated diagnoses, evaluations and prescriptions. Analyzing the journalistic treatment of this matter is important because it involved the interests of the field where the media themselves take part. In addition, they are considered publicly prominent co-constructors of social reality, so they collaborated with the production of narratives about the debate around the Law. This work studies the news frames of the LACS in the Argentine economic press, observes the evolution of these frames throughout the debate and approval of the Law and compares how the newspapers defined the aspects under discussion. The quantitative content analysis identified three frames in tension: 'political and institutional dispute', 'sociocultural public interest' and 'business public interest', which behavior varied according to the newspaper and registered fluctuations throughout the period under study.
\end{abstract}

Keywords

Press, Argentina, regulation, frames, public interest, information.

\title{
Introducción
}

El Poder Legislativo de la Nación (PLN) argentina aprobó la Ley de Servicios de Comunicación Audiovisual (LSCA) $N^{\circ} 26.522$ en octubre de 2009. El proceso de su creación marcó un hito histórico en las políticas públicas de comunicación y les imprimió un cambio de rumbo, pues propuso una nueva noción de interés público a partir de poner en cuestión la histórica relación entre los principios de orden político, sociocultural y económico que había orientado la regulación de los medios audiovisuales de comunicación hasta entonces.

En ese contexto, el propósito de este artículo es analizar el tratamiento de la LSCA en la prensa económica argentina. Concretamente, en $\mathrm{El} \mathrm{Cro-}$ nista Comercial y Ámbito Financiero, dos periódicos de larga tradición en el campo periodístico. Los objetivos específicos son: a) Identificar los frames específicos de la LSCA; b) Analizar el progreso temporal de estos mar- 
cos a lo largo del debate y sanción de la norma; c) Comparar los modos en que ambos diarios presentaron y definieron los asuntos puestos en discusión.

La cobertura del debate, el tratamiento legislativo y la aprobación de la LSCA en este tipo de diarios constituye un objeto apropiado de investigación por varias razones. En primer lugar, estas instituciones mediáticas se vieron involucradas en dicho proceso en su doble rol: tanto como agentes económicos que toman parte en el mercado y en la valorización del capital, cuanto por su carácter de actores políticos en la reproducción ideológica y social. Aun no habiendo sido alcanzados directamente por la norma, esta afectó los intereses de los principales medios de comunicación del país, caracterizados por el alto grado de concentración de la propiedad, tanto de tipo horizontal cuanto vertical y conglomeral (Becerra \& Mastrini, 2018).

El segundo elemento está dado por el hecho de que en la Argentina ha predominado históricamente la lógica comercial en la regulación del funcionamiento de los medios (Mastrini, 2009). Desde el punto de vista empresarial, se trata entonces de un tema de agenda para estos matutinos.

En tercer término, ambos periódicos son considerados aquí actores políticos con intereses particulares y legítimos (Borrat, 1989). Como tales, inciden en la dinámica política y desarrollan representaciones del poder que ejercen y de la sociedad en la cual se desenvuelven (Kircher, 2005). Formadores de opinión, modeladores de la experiencia social y partícipes del campo político, tienen la capacidad de cuestionar o avalar ciertas políticas y, así, nutrir el rol que desempeñan en la esfera pública (Habermas, 1989).

La creación de los encuadres específicos que organizaron el debate en torno a la LSCA resulta novedosa tanto para el campo de estudios de las Políticas de Comunicación cuanto para los análisis de frames mediáticos. Por lo general, las investigaciones filiadas en la economía política crítica dan por sentados los modos en que las disputas de poder se plasman en los discursos de los medios. De modo similar, los análisis de contenido de las coberturas periodísticas, aun cuando abordan debates en torno a la regulación, no suelen relacionar su trabajo con preocupaciones en torno a las políticas públicas (Ali \& Puppis, 2018).

El artículo se organiza como sigue: primero, presenta el procedimiento analítico llevado a cabo para diseñar la investigación. Segundo, se detallan los aspectos relativos al método y al corpus de análisis. Luego, se sistematizan los hallazgos del análisis de contenido. Finalmente, se presentan las conclusiones generales. 


\section{Procedimiento analítico para la creación de los encuadres de la LSCA}

Esta investigación se inscribe conceptualmente en el Framing, entendido como un programa investigativo que comprende integralmente a la comunicación mediática (Koziner, 2015,2017). El trabajo empírico parte de las primeras actividades analíticas que propone este tipo de abordajes (Van Gorp, 2007): detectar y analizar los encuadres ${ }^{1}$ de los medios. En un sentido general, se busca conocer cuál es la estructura de interpretación de la realidad subyacente en el tratamiento periodístico de un caso (Mariño \& López-Rabadán, 2009).

Esta tarea se desarrolla en dos momentos. Primero, se define la noción de encuadre y se desarrolla el procedimiento para su operacionalización. Luego, se diseña una matriz a fin de abordar de manera comprensiva los marcos interpretativos en disputa en torno a la definición de la LSCA y conocer cuáles quedan plasmados como legítimos en las coberturas.

De acuerdo con la clásica conceptualización de Entman (1993, 2004), encuadrar un acontecimiento implica seleccionar ciertos aspectos de aquello que es percibido como la realidad y otorgarles importancia en un texto de forma que se defina una situación problemática, se identifiquen sus causas, se la evalúe moralmente y se proponga un tipo de tratamiento para ella. Así, los frames diagnostican, evalúan y prescriben (Gamson, 1992).

A partir de esta conceptualización, se recurre al planteo epistemológico que llevan a cabo Matthes y Kohring (2008). Ello incluye la recolección de los datos, su procesamiento y la posterior creación de los encuadres propios del caso bajo estudio. El carácter operacional de la definición les permite identificar cuatro funciones que desempeñan los marcos: definir un problema, evaluarlo moralmente, identificar causas y recomendar un tipo de tratamiento. Cada una de estas dimensiones está compuesta por variables de distintos niveles de abstracción y sus indicadores se rastrean en los contenidos de las noticias. Los modos en que esos elementos se combinan dan lugar a ciertos patrones cuya aparición recurrente los convierte en frames.

La conceptualización de los elementos que componen los frames requiere indagar en los rasgos propios del fenómeno e identificar, así, los ejes temáticos que organizan el debate. De modo coherente con dicho procedimiento, en este trabajo se considera necesario el conocimiento de las carac-

1 En este trabajo, frame, encuadre y marco se emplean como sinónimos. 
terísticas centrales de la discusión en torno a la LSCA para la definición de las dimensiones y variables que integran la matriz de análisis.

Para la identificación de los marcos, los autores introducen una técnica de procesamiento computarizado de los datos que permite detectar las variables latentes (Igartua, 2006) en los textos mediante el agrupamiento sistemático de indicadores manifiestos. En contraste con los estudios deductivos, que procuran confirmar o desechar la presencia de encuadres genéricos, este procedimiento permite detectar la emergencia de nuevos frames propios del asunto analizado. El proceso de interpretación y contextualización de tales definiciones de la situación es labor del analista, cuya mirada comprensiva le permite caracterizar el ámbito discursivo en el cual las ideas y argumentos cobran sentido.

\section{Matriz de análisis para la identificación de los encuadres de la LSCA}

A partir de la propuesta de Entman (1993, 2004), a continuación, se conceptualizan las funciones que integran los encuadres sobre la LSCA, así como las variables y los valores que estas pueden cobrar en cada unidad de análisis. De los cuatro frame elements, se hará foco en el primero y en el tercero, dado que su definición involucra el diálogo conceptual entre la perspectiva del Encuadre y las particularidades del caso de acuerdo con los estudios de Políticas de Comunicación. Con todo, en el gráfico 1 se despliegan los elementos, variables y categorías que completan el proceso de operacionalización que se llevó a cabo.

El primer componente involucra una arena o ámbito adonde sucede la acción, un tópico en el que pude clasificarse el acontecimiento y los actores que lo protagonizan mediante sus dichos y/o acciones. Las arenas en torno a la LSCA pueden ser la legislativa, la ejecutiva, la del poder político, la judicial, la empresarial y financiera, la relativa a la sociedad civil y la mediática. Para aquellos ámbitos no considerados en las categorías mencionadas, se incluye la posibilidad de computar otro ámbito.

El tópico alude al issue específico que resume el acontecimiento principal del que trata la noticia, ${ }^{2}$ el cual se observa de modo manifiesto en el tex-

2 Un evento o acontecimiento es un suceso discreto que se encuentra limitado por el tiempo y el espacio (Rogers \& Dearing, 1988). 
to. Tal como consta en el gráfico 1, se identifican siete tópicos en el debate en torno a la LSCA, al cual se suma una octava categoría para otros cuya frecuencia de aparición es baja.

Las fuentes de información y los actores, principales y secundarios, constituyen las últimas cuatro variables que integran la definición del problema. Aunque las categorías diseñadas para unos y otros son las mismas $-\mathrm{y}$, de hecho, pueden coincidir - ambas apuntan a roles diferentes en el relato noticioso. Las fuentes son aquellos a quienes el periodista observa o entrevista y aportan información como miembros o portavoces de grupos con diversos niveles de organización (Gans, 2004). El actor, en cambio, es la persona individual o colectiva involucrada de modo directo en los hechos relatados o presentada como sujeto de la acción. Para este trabajo se elaboró un sistema categorial de 16 opciones desplegadas en el gráfico 1 en función de las recurrencias observadas en una inducción cualitativa a una muestra del corpus.

La evaluación moral constituye la segunda dimensión central para la creación de los encuadres de la LSCA. Estas se expresan como sentencias acerca de lo que debe ser considerado bueno, y los deberes que ello entraña, y acerca de lo que debe ser considerado malo (Schmitt, 2009) para la comunidad. Así, se rastrean en las notas los aspectos más importantes de tres valores constitutivos de la LSCA en torno a los cuales giró el debate público: la sustentabilidad económica del sistema de medios, la diversidad y la libertad de expresión.

Cada uno de estos principios forma parte de uno de los tres tipos de bienestar que debe perseguir un marco regulatorio de los medios audiovisuales que procure promover el interés público: el político, el sociocultural y el económico (Van Cuilenburg \& McQuail, 2003). Aunque el interés público constituye un concepto elástico, vago y complejo (Iosifidis, 2011; Just \& Puppis, 2012; Papathanassopoulos \& Negrine, 2010), se identifican en ella aportes para comprender el cambio de paradigma normativo (Elíades, 2015) que encarna la LSCA para la regulación del sector audiovisual.

En términos abstractos, existe consenso en la evaluación positiva de los tres valores protegidos por la LSCA. Sin embargo, buena parte de las posturas que tomaron los diversos actores involucrados en la discusión pública por la aprobación de la Ley se basaron en distintas miradas sobre ellos y sobre las relaciones que tejen entre sí. Por esa razón, este frame element está integrado por cuatro variables. 


\section{Gráfico 1}

\section{Matriz de análisis de los encuadres de la LSCA}

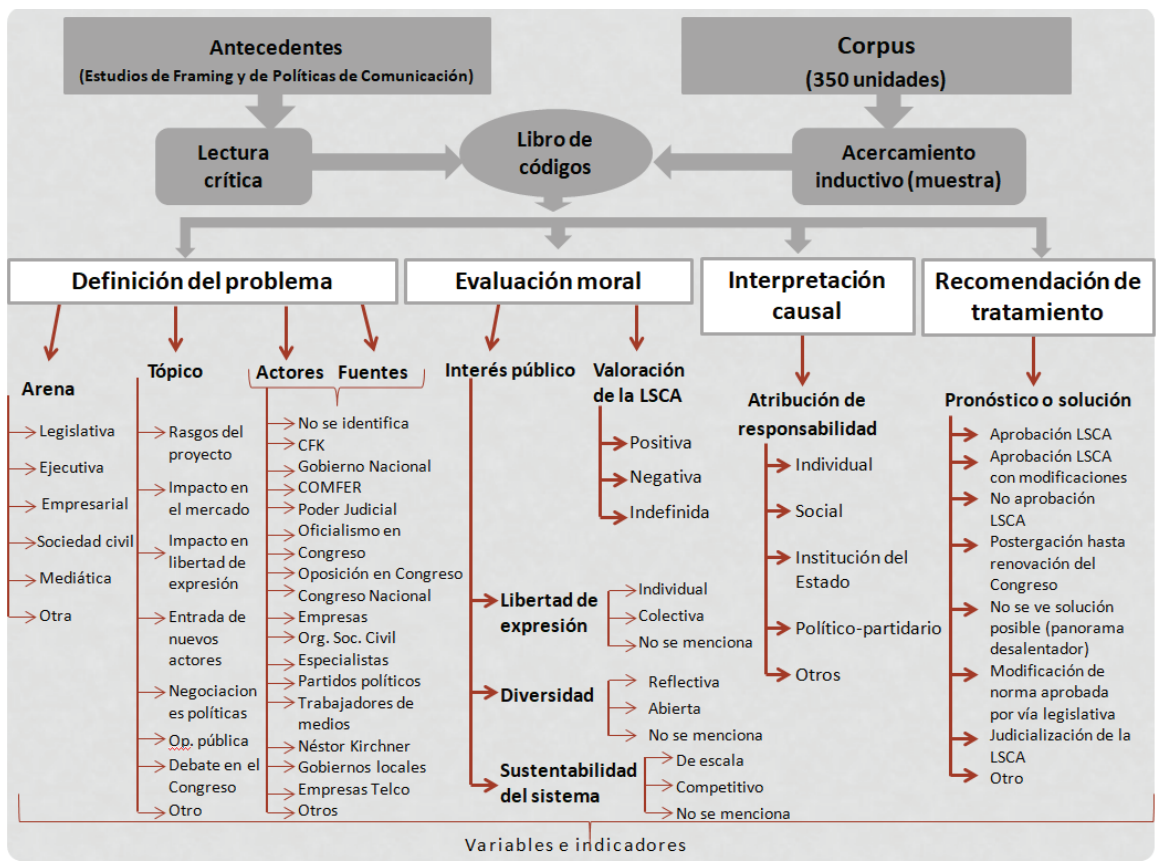

Fuente: Elaboración propia.

En primer lugar, se releva el tipo de valoración de la LSCA. Los juicios positivo y negativo aluden a los casos en que el debate o las implicancias de la aprobación de la norma son valorados como convenientes y/o deseables, por un lado, o inconvenientes y/o no deseables para la sociedad, por otro. El juicio no definido o ambiguo se computa cuando no se pueden identificar con claridad juicios negativos o positivos. También se computa cuando los juicios negativos y positivos se balancean en la misma nota.

En segundo término, se indaga la valoración de la libertad de expresión (Van Cuilenburg \& McQuail, 2003). Se contemplan dos $^{3}$ posibles expresiones de esta variable en los textos: individual y colectiva o social. La prime-

3 Las variables relativas a los valores del interés público contemplan una tercera categoría: "no se menciona", para aquellos casos en que no aparece ningún tipo de referencia al valor. 
ra refiere a la posibilidad o imposibilidad que algunos medios o periodistas específicos tienen de pronunciar libremente sus opiniones e ideas en función de la actuación del Estado, considera que los procesos de desinversión impactan en el ejercicio de la libertad de expresión y/o alude a la libertad de prensa, de modo coherente con la free speech tradition (Loreti \& Lozano, 2014). El carácter social o colectivo se computa cuando se menciona la importancia de una mayor participación de variados actores sociales en los medios, la necesidad de poner límites a las fuerzas del mercado y/o la discusión plural del proyecto de Ley, en sintonía con la concepción propuesta por la Corte Interamericana de Derechos Humanos (1985).

La segunda variable apunta al pluralismo y a la diversidad del sistema de medios y contempla dos categorías: el principio de diversidad reflectiva apunta a que la variedad de opiniones e issues que expresen los medios debe responder a aquello que prefieren consumir las audiencias. La diversidad abierta, en cambio, refiere a que los medios deben representar lo más acabadamente posible el abanico de voces, asuntos y opiniones que circulan socialmente, más allá de lo que demanden las audiencias (Van der Wurff \& Van Cuilenburg, 2001).

La última variable que integra la definición de interés público alude a la sustentabilidad del sistema de medios. Esta puede asumir dos valores: economías de escala o mercado competitivo. Resulta difícil encontrar estos valores explícitamente expresados en los textos, dado el carácter técnico que revisten, por lo que se observa la presencia de argumentos afines a estos principios. En el primer caso, se considera que las medidas que buscan limitar la concentración de la propiedad mellan la rentabilidad de los medios o, más aun, la sustentabilidad de las empresas (Llorens-Maluquer, 2001). De acuerdo con el segundo valor, la apertura del mercado a nuevos actores impacta positivamente en su funcionamiento. Se lo computa, asimismo, cuando la concentración es caracterizada como un problema de orden económico que erosiona la competencia y la eficiencia del mercado (Doyle, 2002).

\section{Corpus, codificación y fiabilidad}

El corpus del estudio está conformado por todos los artículos periodísticos publicados acerca de la LSCA en El Cronista Comercial y Ámbito Financiero a partir del primer día del mes en que se presentó el anteproyecto de ley - el 1 de marzo de 2009 - y el último del mes en que se aprobó la norma - el 31 de octubre - . En total, se recabaron 350 unidades. 
Todas las unidades sometidas al análisis de contenido cuantitativo fueron seleccionadas y codificadas con arreglo a idénticas normas detalladas y manifiestas (Colle, 2011; Igartua, 2006). La inmersión cualitativa (Neuendorf, 2002) que se llevó a cabo a una muestra de 50 unidades - el 14,3\% ${ }^{4} \mathrm{de}$ las notas - permitió identificar las "variables críticas" del estudio (Igartua, 2006) en diálogo con los rasgos de la LSCA y de los diarios.

La calidad de los datos obtenidos mediante esta técnica se vincula con tres aspectos: una precisa conceptualización y operacionalización de las variables, la instrucción de quienes llevarán a cabo la codificación y los niveles de "fiabilidad intercodificadores". Este mide los niveles de acuerdo entre los datos recogidos por distintos investigadores que abordaron los mismos contenidos con idéntico libro de códigos (Igartua \& Humanes, 2004).

En este trabajo, cuatro codificadores participaron de la recolección de los datos. Para medir el grado de acuerdo entre las variables se utilizaron dos coeficientes: Alfa de Krippendorff $(\alpha)$ y Kappa de Cohen $(\varkappa) .{ }^{5}$ Todos los cálculos de los coeficientes de fiabilidad (Krippendorff, 2004) resultaron por arriba de 0.85 .

\section{Análisis y resultados. Los encuadres de la LSCA}

Esta fase del trabajo procura conformar los frames mediáticos de la LSCA en los diarios económicos, analizar su evolución y comparar las coberturas.

En primer término, se lleva a cabo un análisis estadístico de conglomerados o clusters. Este método busca agrupar las unidades en conjuntos lo más semejantes posible. El procesamiento identifica, a través de algoritmos matemáticos, conjuntos de características afines en un universo de casos que aparenta ser heterogéneo (Vilà-Baños et al., 2014). Como resultado, cada caso es asignado a un grupo específico que, en última instancia, conforma un encuadre.

Del procesamiento de los datos surge una solución óptima de tres clusters o frames. La lectura interpretativa de cada conjunto permitió denominarlos 'disputa política e institucional', 'interés público empresario' e 'interés público sociocultural'.

El primer conglomerado agrupa el 55,4\% de los casos del universo. El encuadre refiere fundamentalmente a las disputas y negociaciones que se

4 Si bien existe acuerdo entre los teóricos del análisis de contenido respecto de que la muestra debe ser del 10\% de las unidades, se recomienda que esta no sea menor a 50 unidades en total (Igartua, 2006).

5 A fin de conocer el Alfa de Krippendorff $(\alpha)$ la versión de SPSS utilizada fue macro 3.0, en sintonía con lo propuesto por Hayes y Krippendorff (2007). 
producen entre las distintas fuerzas políticas en el marco del PLN, ámbito en el cual se debatió el proyecto normativo. La tabla 1 sintetiza las categorías más importantes de este encuadre.

Se observa que la mayor parte de las notas que citan dos fuentes, enfrentan posturas opuestas ante la LSCA, lo cual aporta al componente de conflicto. Idéntico comportamiento se evidencia respecto de los actores protagonistas de las noticias.

\section{Tabla 1}

Frame elements y variables de la 'Disputa política e institucional'

\begin{tabular}{|c|c|c|}
\hline \multicolumn{2}{|c|}{ Elementos y variables } & Categorías \\
\hline \multirow{6}{*}{ 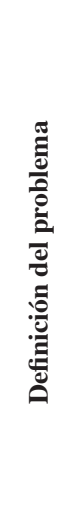 } & Arena & Legislativa \\
\hline & Tópico & $\begin{array}{l}\text { Tratamiento del proyecto en el Congreso } \\
\text { Negociaciones políticas }\end{array}$ \\
\hline & Fuente principal & $\begin{array}{l}\text { Oposición política en el PLN } \\
\text { Oficialismo político en el PLN }\end{array}$ \\
\hline & Fuente secundaria & $\begin{array}{l}\text { No hay } \\
\text { Oposición política en el PLN } \\
\text { Oficialismo político en el PLN }\end{array}$ \\
\hline & Actor principal & $\begin{array}{l}\text { Oposición política en el PLN } \\
\text { Oficialismo político en el PLN }\end{array}$ \\
\hline & Actor secundario & $\begin{array}{l}\text { Oposición política en el PLN } \\
\text { Gobierno de la Nación }\end{array}$ \\
\hline 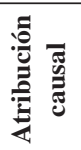 & $\begin{array}{l}\text { Atribución de } \\
\text { responsabilidad }\end{array}$ & $\begin{array}{l}\text { Institucional } \\
\text { Política }\end{array}$ \\
\hline \multirow{4}{*}{ 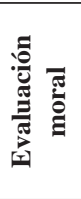 } & Valoración LSCA & No definida o ambigua \\
\hline & Libertad de expresión & - \\
\hline & Diversidad & - \\
\hline & Sustentabilidad & - \\
\hline 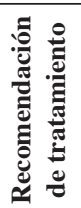 & Solución propuesta & $\begin{array}{l}\text { No se hace mención } \\
\text { Aprobación de la LSCA } \\
\text { Aprobación de la LSCA con modificaciones. }\end{array}$ \\
\hline
\end{tabular}

Fuente: Elaboración propia. 
El alto grado de institucionalización en el marco de este encuadre se relaciona con el "sesgo de trastorno de autoridad" (authority-disorder bias) (Bennett, 2012, p. 63), tendencia que puede combinarse con episodios noticiosos personalizados, dramatizados o fragmentados. Esto porque su rasgo central consiste en el alto énfasis de las noticias en las acciones de las autoridades - si toman las riendas o pierden el control, si ganan, pierden o negocian - y en establecer "si la situación en cuestión parece moverse en una dirección más ordenada y tranquilizadora o desordenada y perturbadora". Así, no son tan importantes los detalles del debate de la política pública como los aspectos dramáticos y controversiales que lo rodean. La valoración indefinida de la iniciativa debatida en el Congreso y la nula frecuencia de aparición de los valores que integran los tipos de bienestar incluidos en la noción de 'interés público' son coherentes con los rasgos generales de este frame.

El nivel de generalidad de este encuadre permite vincularlo con la definición de "guion" (script) que propone Entman (2004). Es decir, como un modelo estandarizado de procesamiento de información que los periodistas utilizan para tratar los asuntos de interés público. La principal diferencia entre los "guiones" y los "encuadres" radica en que los primeros encauzan las historias, mientras que los segundos promueven definiciones de la situación que conllevan evaluaciones morales y proponen un tipo de tratamiento para los acontecimientos relatados (Entman, 2004, pp. 6-7). En efecto, la disputa en torno a la LSCA y sus implicancias político-partidarias cobran protagonismo en detrimento la evaluación moral de la medida y los pronósticos en torno a ella.

Tabla 2

Frame elements y variables del 'Interés público empresarial'

\begin{tabular}{|c|c|c|}
\hline \multicolumn{2}{|c|}{ Elementos y variables } & Categorías predominantes \\
\hline \multirow{5}{*}{ 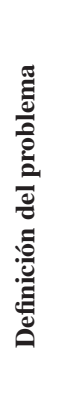 } & Arena & $\begin{array}{l}\text { Empresarial y financiera } \\
\text { Poder político }\end{array}$ \\
\hline & Tópico principal & $\begin{array}{l}\text { Impacto de la norma en el mercado mediático Características } \\
\text { del proyecto / de la LSCA }\end{array}$ \\
\hline & Fuente principal & $\begin{array}{l}\text { Empresas de comunicación } \\
\text { Gobierno de la Nación }\end{array}$ \\
\hline & Fuente secundaria & $\begin{array}{l}\text { Empresas de comunicación } \\
\text { Gobierno de la Nación }\end{array}$ \\
\hline & Actor principal & $\begin{array}{l}\text { Gobierno de la Nación } \\
\text { Empresas de comunicación }\end{array}$ \\
\hline
\end{tabular}




\begin{tabular}{|c|c|c|}
\hline & Actor secundario & $\begin{array}{l}\text { Empresas de comunicación } \\
\text { Gobierno de la Nación }\end{array}$ \\
\hline 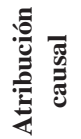 & $\begin{array}{l}\text { Atribución de } \\
\text { responsabilidad }\end{array}$ & $\begin{array}{l}\text { Política } \\
\text { Individual }\end{array}$ \\
\hline \multirow{4}{*}{ 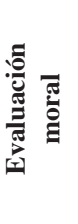 } & Valoración LSCA & $\begin{array}{l}\text { Negativa } \\
\text { Positiva }\end{array}$ \\
\hline & Libertad de expresión & Individual \\
\hline & Diversidad & Reflectiva \\
\hline & Sustentabilidad & Economías de escala \\
\hline 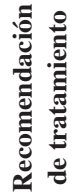 & Solución propuesta & $\begin{array}{l}\text { No se vislumbra solución } \\
\text { Judicialización de la Ley }\end{array}$ \\
\hline
\end{tabular}

Fuente: Elaboración propia.

El segundo cluster reúne el 25,5\% de los casos. Se lo denomina 'Interés público empresario', en tanto fija los parámetros para el debate en función de argumentos que privilegian los criterios económicos por sobre los políticos y culturales. Los valores priorizados se corresponden con una mirada contradictoria con los principios sostenidos por el texto normativo. La tabla 2 sintetiza las categorías más importantes del encuadre.

La acción noticiosa evoca situaciones que giran alrededor de los rasgos del texto de la LSCA dirigidos a regular el funcionamiento del mercado de medios, así como de las consecuencias económico-financieras que la norma tendría para las dinámicas de dicho mercado. Frecuentemente, el protagonismo del Gobierno nacional está asociado a causas - o responsabilidades - de tipo político-partidarias o de carácter individual mucho más que a su rol institucional. Ello da cuenta de una tendencia de los periódicos a la "personalización" (Bennett, 2012), es decir, a desarrollar relatos más centrados en los actores que en de los eventos en sí. En contrapartida, se sobrevaloran los motivos y las intenciones personales o político-partidarias en las atribuciones de responsabilidad, al tiempo que se subestima el papel que desempeña el contexto (Iyengar, 1991).

La mirada individual de la libertad de expresión, la diversidad de tipo reflectiva y las economías de escala no aparecen explícitamente menciona- 
das en los textos. En cambio, se menciona la libertad de prensa y se advierte la potencial amenaza para su ejercicio en el marco del sistema democrático que significaría la LSCA. Además, se sostiene que la reestructuración del sistema que impondrían los límites a la propiedad de licencias o la obligación de producir contenidos locales, afectaría la pluralidad de voces del sector privado comercial y se considera que el deber de desinvertir acarrearía un serio peligro para la sustentabilidad o la seguridad jurídica de las empresas o grupos mediáticos que superan los límites dispuestos por la Ley.

Tabla 3

Frame elements y variables del 'Interés público sociocultural'

\begin{tabular}{|c|c|c|}
\hline \multicolumn{2}{|r|}{ Elementos y variables } & Categorías predominantes \\
\hline \multirow{6}{*}{ 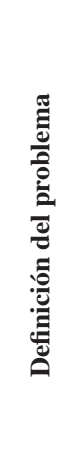 } & Arena & $\begin{array}{l}\text { De la sociedad civil } \\
\text { Legislativa }\end{array}$ \\
\hline & Tópico principal & $\begin{array}{l}\text { Características del proyecto/de la LSCA Impacto en } \\
\text { la libertad de prensa y/o expresión }\end{array}$ \\
\hline & Fuente principal & $\begin{array}{l}\text { Organizaciones de la sociedad civil } \\
\text { Especialistas }\end{array}$ \\
\hline & Fuente secundaria & No hay \\
\hline & Actor principal & $\begin{array}{l}\text { Organizaciones de la sociedad civil } \\
\text { Gobierno de la Nación }\end{array}$ \\
\hline & Actor secundario & $\begin{array}{l}\text { Gobierno del a Nación } \\
\text { Organizaciones de la sociedad civil }\end{array}$ \\
\hline 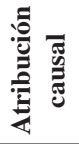 & Atribución de responsabilidad & $\begin{array}{l}\text { Institucional } \\
\text { Política }\end{array}$ \\
\hline \multirow{4}{*}{ 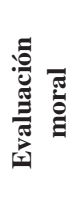 } & Valoración LSCA & $\begin{array}{l}\text { No definida } \\
\text { Positiva }\end{array}$ \\
\hline & Libertad de expresión & Colectiva \\
\hline & Diversidad & Abierta \\
\hline & Sustentabilidad & Mercado competitivo \\
\hline 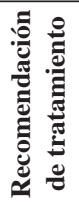 & Solución propuesta & Aprobación de la LSCA \\
\hline
\end{tabular}

Fuente: Elaboración propia. 
El último conglomerado agrupa el 19,1\% de los casos del universo. El frame representa la mirada en la cual se priorizan los valores de tipo sociocultural y político por sobre los del bienestar económico y tiende a coincidir con las posturas favorables a la LSCA. La tabla 3 sintetiza las categorías más importantes del frame.

Los actores estatales y los pertenecientes a la sociedad civil adquieren mayor protagonismo como agentes capaces de incidir en el desarrollo de los eventos que se relatan. La evaluación moral que promueve este encuadre es coherente, en términos generales, con el enfoque de interés público protegido por el texto de la LSCA. Así, la mirada colectiva de la libertad de expresión se combina con un enfoque abierto de la diversidad y el pluralismo y con una comprensión del mercado mediático en términos competitivos. No obstante, esta valoración del interés público no deriva necesariamente en un juicio positivo del proyecto de ley de SCA, pues conviven miradas contradictorias. De ello se infiere que el argumento general promovido por este frame no puede emparentarse linealmente con el apoyo de la iniciativa legislativa, aunque coincida, en términos generales. Sucede que algunas posturas críticas a la norma se fundan, justamente, en los mismos valores que esta procura proteger.

El segundo objetivo específico del trabajo se proponía analizar la evolución temporal de los encuadres a lo largo del debate y la sanción de la norma. Esta es representada en el gráfico 2, según cuatro etapas de desarrollo del caso: 1) de debate público: a partir de la presentación del anteproyecto de ley (18 de marzo) hasta su envío al Congreso de la Nación (28 de agosto); 2) tratamiento en la Cámara Baja: entre el 31 de agosto y el 18 de septiembre, un día después de haber obtenido media sanción; 3) tratamiento en el Senado: desde el 21 de septiembre hasta el 9 de octubre, un día antes de convertirse en Ley; ${ }^{6}$ 4) de repercusiones: entre el 12 y el 30 de octubre de 2009.

Del gráfico se desprende que los tres encuadres están presentes durante las 36 semanas estudiadas, aunque el comportamiento de cada uno varía de modo singular. La disputa política e institucional fluctúa de modo concomitante con la frecuencia general del caso. En tanto, el interés público empresario presenta una menor frecuencia global, que decrece conforme avanzan las semanas. El encuadre interés público sociocultural, finalmente, es el de

6 La LSCA fue aprobada durante la madrugada del sábado 10 de octubre, día en que no se editan los diarios económicos. Por esa razón, la fase se cierra el viernes 9, cuando ambos periódicos adelantan que el proyecto se convertiría en Ley. 
menor nivel de aparición en términos generales, aunque se mantiene estable durante las fases de mayor relevancia del caso.

\section{Gráfico 2}

Evolución temporal de los frames según etapas del período (1 de marzo-31 de octubre de 2009). El Cronista Comercial y Ámbito Financiero

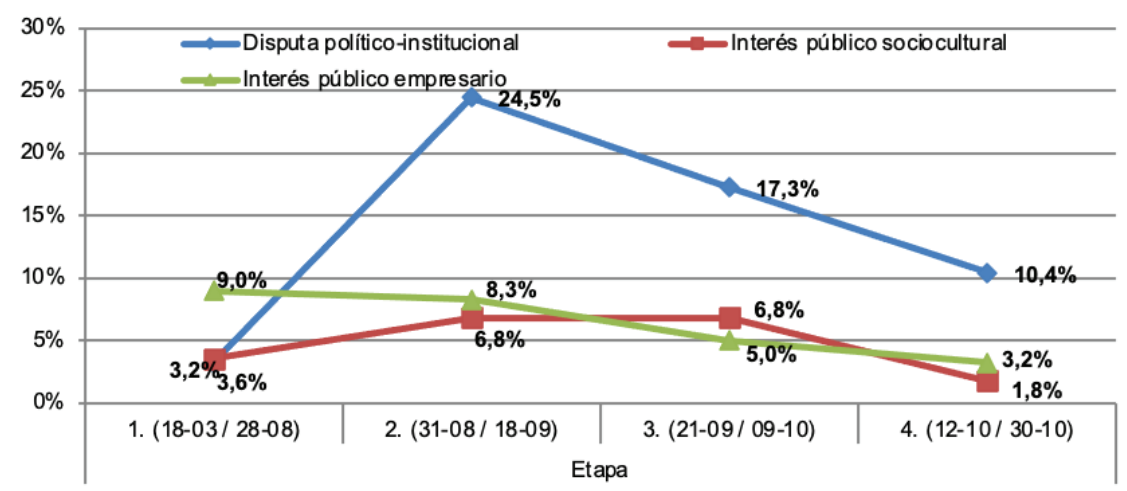

Fuente: Koziner, 2017.

El último objetivo específico apuntaba a comparar el tratamiento que ambos diarios le dieron al caso. En este sentido, los datos que arroja el gráfico 3 son elocuentes: aunque los tres encuadres están presentes en los dos periódicos, en Ámbito Financiero siete de cada diez notas son encuadradas como una disputa política e institucional, reservando un espacio sensiblemente menor a los encuadres específicos de la LSCA. El Cronista Comercial, en cambio, no solo ofrece una cobertura globalmente más abundante sino también más heterogénea respecto de los modos de encuadrar el debate por la Ley.

En estas diferencias es posible observar las huellas del perfil editorial de los diarios. En Ámbito Financiero predominó ampliamente el componente controversial que suele caracterizar todas las coberturas de asuntos políticos y se prestó menor atención a los aspectos específicos del caso. Aun cuando en El Cronista Comercial también predominó el frame disputa política e institucional, los otros dos también fueron relevantes. Especialmente, el interés público empresarial, que se destacó en las notas del género argumentativo y, dentro de estas, en los editoriales. 


\section{Gráfico 3}

Presencia de los encuadres 'Disputa política e institucional', 'Interés público empresario' e 'Interés público sociocultural' (1 de marzo-31de octubre de 2009). El Cronista Comercial y Ámbito Financiero

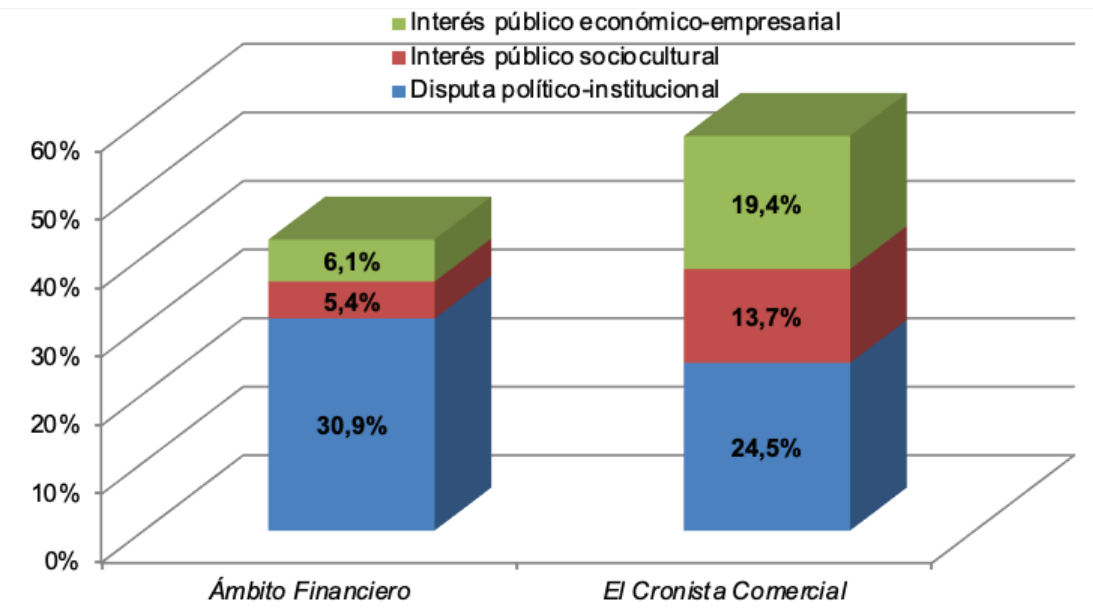

Fuente: Koziner, 2017.

\section{Discusión y conclusiones}

El presente artículo se propuso analizar el tratamiento que los diarios económicos argentinos hicieron del derrotero que recorrió el proceso de debate y sanción de la LSCA, asumiendo que el asunto reviste sumo interés para este sector de los medios, tanto por integrar el campo de los actores alcanzados por la norma, cuanto por constituir un asunto con consecuencias económicas para buena parte del sector.

Tres fueron los marcos hallados: 'disputa política e institucional', 'interés público empresario' e 'interés público sociocultural'. La disputa política e institucional podría pensarse menos como un encuadre y más como un "guion" (script), en el sentido definido por Entman (2004, p. 6).

El interés público empresarial logra imprimir una mirada negativa de la LSCA, mientras el interés público sociocultural sintetiza una postura emparentada con los argumentos sostenidos por la iniciativa, pero no totalmente alineada 
con ella. No fue casual que este encuadre hubiera sido el menos relevante, habida cuenta de que, coherentemente con lo sostenido por la Ley, propuso una visión renovada acerca de cómo debía evaluarse moralmente la regulación en comunicación audiovisual. Así, resultó contradictoria con la tradición normativa argentina del campo, favorable a la lógica comercial y privada de funcionamiento.

La evolución temporal de los tres frames no fue homogénea; tampoco la relevancia que cobraron en la superficie de los periódicos. El comportamiento del encuadre 'disputa política e institucional' fluctuó de modo concomitante con la frecuencia general de la cobertura de la Ley, por lo que estuvo fuertemente vinculado con el devenir de los acontecimientos. Se infiere, así, que predominó un abordaje fundamentalmente episódico, centrado en eventos concretos con un alto grado de controversia y dramatismo.

La interpretación del comportamiento de los encuadres en cada diario puede ponerse en relación con dos aspectos centrales: el modo en que la iniciativa regulatoria dialogó con la historia y situación de cada uno y las tradiciones editoriales del periodismo económico en las cuales podrían filiarse.

La LSCA retomó algunos puntos de un reclamo histórico contra Clarín por parte de Julio Ramos, creador de Ámbito Financiero, por la conformación y exponencial crecimiento del grupo empresario a partir de la década de los noventa (Ramos, 1993; Ulanovsky, 2011). No obstante, la postura crítica hacia este tipo de procesos, incluso cuando se afirmaba que impactaban sobre la libertad de expresión, se fundaban en una concepción de interés público ligeramente divergente a la propuesta por la LSCA. Desde aquel punto de vista, la libre competencia y las reglas antimonopólicas son los valores centrales para cualquier política de medios, por lo que la intervención estatal debe limitarse a garantizar esas condiciones.

En términos generales, Ámbito se ubicó como un observador externo de un debate político que lo involucraba solo indirectamente. Los encuadres específicos de la LSCA podrían considerarse más como filtraciones de los modos de definir la medida por parte de las fuentes y de los actores que participaron del debate y menos como definiciones compartidas por la línea editorial del periódico.

La estructura de propiedad de El Cronista Comercial colocó a esta institución periodística en una situación particular, pues su propietario, Francisco De Narváez, se encontraba en infracción a la Ley por ser diputado nacional y licenciatario del canal de televisión América TV. Su postura institucional fue compatible con la definición propuesta por el interés público empresario y quedó expresada, en buena medida, en los editoriales dedica- 
dos al asunto. En línea con ello, se observó una mayor presencia del sector empresario en sus páginas, como fuente y como protagonista de la acción relatada. La cobertura de El Cronista resultó más heterogénea que la de su competidor en términos de los frames presentes en el tratamiento informativo y de las fuentes y actores involucrados.

Amén de las diferencias, la centralidad de los actores oficiales, la jerarquización de las arenas legislativa y ejecutiva por sobre los debates en la sociedad civil y la controversia como eje central de las noticias fueron elementos comunes a los dos diarios.

Finalmente, en ambos periódicos es posible hallar huellas de los dos modelos originarios del periodismo económico: el estadounidense, en $E l$ Cronista y el británico, en Ámbito. En este último, la información se mezcla con el análisis, los comentarios y las predicciones. Además, se caracteriza por el uso frecuente del off the record. El estadounidense, en cambio, suele separar con mayor claridad la información de la opinión y la primera está acompañada de mayor cantidad de fuentes, generalmente institucionales. La opinión suele articularse con voces externas y expertas en el campo.

Además de los hallazgos específicos a los que ha arribado este trabajo, interesa recalcar un factor en relación con los encuadres: la creación, cristalización y circulación de estos patrones organizadores de los sentidos de las noticias son el resultado de dinámicas en las cuales interactúan diversos factores - culturales, económicos, políticos - que desbordan a los textos, aunque se plasman en ellos. Los encuadres son principios persistentes que integran todas las instancias del proceso comunicacional y que deben ser compartidos en algún nivel por las sociedades en las cuales se desarrollan para ser significativos y comunicables. Es preciso considerar, entonces, que, aunque los frames del tratamiento de la LSCA no hayan nacido en la prensa analizada, fueron claves poderosas en la organización del sentido de sus contenidos.

\section{Apoyos y soporte financiero de la investigación}

Entidad: Consejo Nacional de Investigaciones Científicas y Técnicas (CONICET)

País: Argentina

Ciudad: Ciudad Autónoma de Buenos Aires

Proyecto subvencionado: Beca doctoral PG 11 2012-2017 


\section{Bibliografía}

Ali, C., \& Puppis, M. (2018). When the watchdog neither barks nor bites: Communication as a power resource in media policy and regulation. Communication Theory, 28(3), 270-291. https://doi.org/10.1093/ct/qtx003

Becerra, M., \& Mastrini, G. (2018). Políticas de medios del kirchnerismo. Análisis de las políticas de comunicación 2003- 2015 y agenda pendiente. En Medios en guerra $\left(1^{\circ}\right)$. Biblos.

Bennett, W.L. (2012). News. The Politics of Illusion $\left(9^{\circ}\right)$. Pearson Education, Inc.

Borrat, H. (1989). El periódico, actor político $\left(1^{\circ}\right)$. Gustavo Gili.

Colle, R. (2011). El análisis de contenido de las comunicaciones. En Colección Cuadernos Artesanos de Latina ( $9^{\circ}$, Vols. 11-12-13). Sociedad Latina de Comunicación Social. https://bit.ly/3bTi77v

Opinión Consultiva OC-5/85: La Colegiación Obligatoria de periodistas (arts. 13 y 29 de la Convención Americana de Derechos Humanos) (1985). https:// bit.ly/2LFZZTX

Doyle, G. (2002). Media Ownership (1). Sage Publications.

Elíades, A. (2015). Derecho a la Comunicación y 30 años de democracia $\left(1^{\circ}\right)$. EDULP.

Entman, R.M. (1993). Framing: Toward Clarification of a Fractured Paradigm. Journal of Communication, 43(4), 51-58. https://doi.org/10.1111/j.1460-2466. 1993.tb01304.x

Entman, R.M. (2004). Projections of power: Framing news, public opinion, and US foreign policy. University of Chicago Press.

Gamson, W.A. (1992). The social psychology of collective action. En A.D. Morris \& C. McClurg Mueller (Eds.), Frontiers in social movement theory (pp. 53-76). Yale University Press.

Gans, H.J. (2004). Deciding What's News: A Study of CBS Evening News, NBC Nightly News, Newsweek, and Time $\left(2^{\circ}\right)$. Northwestern University Press.

Habermas, J. (1989). The Structural Transformation of the Public Sphere. Mass.

Hayes, A. F., \& Krippendorff, K. (2007). Answering the call for a standard reliability measure for coding data. Communication Methods and Measures, 1, 7789. http://www.afhayes.com/spss-sas-and-mplus-macros-and-code.html

Igartua, J.J. (2006). El análisis de contenido. En Métodos cuantitativos de investigación en comunicación ( $1^{\circ}$, pp. 175-229). Bosch.

Igartua, J.J., \& Humanes, M. L. (2004). El método científico aplicado a la investigación en comunicación social. Portal de la Comunicación InCom-UAB. Aula abierta. Lecciones básicas, 1986, 18. https://bit.ly/3ixjREQ 
Iosifidis, P. (2011). Global Media and Communication Policy. En Journal of Broadcasting \& Electronic Media $\left(1^{\circ}\right)$. Palgrave Macmillan. https://doi. org/10.1177/1742766507082572

Iyengar, S. (1991). Is Anyone Responsible? How Television Frames Political Issues $\left(1^{\circ}\right)$. The University of Chicago Press. https://bit.ly/2LOLvkA

Just, N., \& Puppis, M. (2012). Trends in Communication Policy Research. New Theories, Methods and Subjects $\left(1^{\circ}\right)$. Intellect.

Kircher, M. (2005). Prensa escrita: actor social y politico, espacio de producción cultural y fuente de información histórica. Revista de Historia, 10, 115122. https://bit.ly/2KzSmhe

Koziner, N. (2015). El Framing: un programa de investigación para el estudio de las comunicaciones mediáticas. Animus. Revista Interamericana de Comunicação Midiática, 14(28), 22-45. https://doi.org/10.5902/21754977

Koziner, N. (2017). La prensa económica y el debate sobre los medios. Análisis de la cobertura informativa del proceso de discusión de la Ley de Servicios de Comunicación Audiovisual en Ámbito Financiero y El Cronista Comercial (marzo-octubre de 2009). Universidad Nacional de Quilmes.

Krippendorff, K. (2004). Reliability in Content Analysis: Some Common Misconceptions and Recommendations. Human Communication Research, 30(3), 411433. https://doi.org/http://dx.doi.org/10.1111/j.1468-2958.2004.tb00738.x

Llorens-Maluquer, C. (2001). Concentración de empresas de comunicación y el pluralismo: la acción de la Unión Europea. Universitat Autònoma de Barcelona. https://bit.ly/2LOM264

Loreti, D., \& Lozano, L. (2014). El derecho a comunicar. Los conflictos en torno a la libertad de expresión en las sociedades contemporáneas $\left(1^{\circ}\right)$. Siglo XXI Editores.

Mariño, M.V., \& López-Rabadán, P. (2009). Resultados actuales de la investigación sobre framing: sólido avance internacional y arranque de la especialidad en España. Zer, 14(26), 13-34. https://bit.ly/2XYepBq

Matthes, J., \& Kohring, M. (2008). The Content Analysis of Media Frames: Toward Improving Reliability and Validity. Journal of Communication, 58(2), 258-279. https://doi.org/10.1111/j.1460-2466.2008.00384.x

Neuendorf, K. (2002). The content analysis guidebook $\left(1^{\circ}\right)$. Sage.

Papathanassopoulos, S., \& Negrine, R. (2010). Communications Policy. Theories and Issues $\left(1^{\circ}\right)$. Palgrave Macmillan.

Ramos, J.A. (1993). Los cerrojos a la prensa $\left(1^{\circ}\right)$. Editorial Amfin. 
Rogers, E.M., \& Dearing, J.W. (1988). Agenda-setting research: Where has it been, where is it going? En J. A. Anderson (Ed.), Communication yearbook 11 $\left(1^{\circ}\right.$, pp. 555-594). Sage.

Schmitt, C. (2009). El concepto de lo político. Alianza Editorial. https://doi.org/10. 1007/s13398-014-0173-7.2

Ulanovsky, C. (2011). Paren las rotativas. Diarios, revistas y periodistas (19702000) ( $2^{\circ}$ edición). Emecé.

Van Cuilenburg, J., \& McQuail, D. (2003). Cambios en el paradigma de política de medios. Hacia un nuevo paradigma de políticas de comunicación. European Journal of Communication, 18(2), 181-207.

Van der Wurff, R., \& Van Cuilenburg, J. (2001). Impact of Moderate and Ruinous Competition on Diversity: The Dutch Television Market. Journal of Media Economics, 14(4), 213-229. https://doi.org/10.1207/S153 27736ME1404

Van Gorp, B. (2007). The Constructionist Approach to Framing: Bringing Culture Back In. Journal of Communication, 57(1), 60-78. https://doi. org/10.1111/j.1460-2466.2006.00329.x

Vilà-Baños, R., Rubio-Hurtado, M.J., Berlanga-Silvente, V., \& Torrado-Fonseca, M. (2014). Cómo aplicar un cluster jerárquico en SPSS. REIRE. Revista d'Innovació i Recerca en Educació, 7(1), 113-127. https://doi.org/10.13 44/reire2014.7.1717

Fecha de envío:2020/10/31; Fecha de aceptación: 2021/01/18;

Fecha de publicación: 2021/03/01 